\title{
用表面增强拉曼散射探测 $\mathrm{Bi}^{3+}$ 离子 对表面络合物的作用 ${ }^{*}$
}

\author{
李特新 刘玉龙 莫育俊 \\ (中国科学院物理研究所, 北京 100080) \\ 雷洁苟增光 \\ (陕西师范大学物理系, 西安 710062)
}

\section{关链调 表面增强拉曼散射、Bi ${ }^{3+} 、\left[\mathrm{Ag}(\mathrm{SCN})_{2}\right]^{-}$}

络合物在金属表面上的形成是一个基本而又重要的过程. 对表面络合物相应结 构的阐 述,无论对于固液界面性质的研究,还是固体表面化学反应、表面相变及催化机理等方面的研 究, 都有着广泛的实际意义。表面增强拉曼散射 (Surface-enhanced raman scattering, 简 马 SERS) 自发现以来 ${ }^{[1]}$, 由于其高灵敏度和易检测等优点, 已经成为研究和实时监测表面上 分子振动光谱的有力工具 ${ }^{[2]}$. 用 SERS 研究络合物及金属表面形成的络合物也有一些报 道 ${ }^{[3,4]}$.

在本文中,选用 $\mathrm{SCN}^{-} / \mathrm{Ag}$ SERS 系统. $\mathrm{SCN}^{-}$离子具有“准卤离子”性质,它的构型是线 型(点群 $C_{\infty}$ ) 或略有弯曲 (点群 $C_{s}$ ). $\mathrm{SCN}^{-} / \mathrm{Ag}$ 的四个 SERS 谱带: 205, 445, 736 和 2122 $\mathrm{cm}^{-1}$, 根据文献 [5], 被分别指认为 $\mathrm{Ag}^{-\mathrm{SCN}^{-}}$的伸张振动、 $\mathrm{SCN}^{-}$弯曲振动、 $\mathrm{C}-\mathrm{S}$ 伸张振 动和 $\mathrm{C} \equiv \mathrm{N}$ 伸张振动, 而且从 $\mathrm{C} \equiv \mathrm{N}$ 伸张振动峰的位置可以确定 $\mathrm{SCN}^{-}$离子是以 $\mathrm{N}$ 原子 还是以 $\mathrm{S}$ 原子与银表面键接 ${ }^{[5,6]}$.

我们对 $\mathrm{SCN}^{-} / \mathrm{Ag}$ 系统 $\mathrm{SERS}$ 谱的测量, 得到的 $\mathrm{C} \equiv \mathrm{N}$ 伸张振动峰位于 $2122 \mathrm{~cm}^{-1}$, 即 在银表面形成了以 $S$ 原子与银键接的表面络合物离子 $\left[\mathrm{Ag}(\mathrm{SCN})_{2}\right]^{-[5-7)}$, 通过监测该系统 $\mathrm{SE}$ RS 谱对 $\mathrm{Bi}^{3+}$ 离子加人的响应, 发现 SERS 谱强度随加人 $\mathrm{Bi}^{3+}$ 离子浓度的增加而降低, 而 且峰宽略变窄 $\left(2122 \mathrm{~cm}^{-1}\right.$ 峰较为明显), 这些现象归因为 $\mathrm{Bi}^{3+}$ 离子对 $\left[\mathrm{Ag}(\mathrm{SCN})_{2}\right]^{-}$离子的 作用.

\section{一、实验}

1. 溶液配制 将 $2.33 \mathrm{~g} \mathrm{Bi}_{2} \mathrm{O}_{3}$ 溶于 $100 \mathrm{ml} 1: 1$ 的 $\mathrm{HNO}_{3}$ 水溶液中, 得到 $\mathrm{Bi}^{3+}$ 离子 浓度为 $0.1 \mathrm{~mol} / \mathrm{L}$ 的溶液, 依次稀释到实验所需的浓度: $8.0 \times 10^{-4} 、 1.6 \times 10^{-4} 、 3.2 \times 10^{-5}$ 、 $6.4 \times 10^{-6} 、 1.3 \times 10^{-6} \mathrm{~mol} / \mathrm{L}$ ，各溶液中对应的 $\mathrm{NO}_{3}^{-}$离子浓度分别为 $2 \times 10^{-2} 、 4 \times 10^{-3}$ 、 $8 \times 10^{-4} 、 1.6 \times 10^{-4} 、 3.2 \times 10^{-5} \mathrm{~mol} / \mathrm{L}$. 还配制了 $0.05 \mathrm{~mol} / \mathrm{L}$ 的 $\mathrm{KSCN}$ 溶液和 $0.02 \mathrm{~mol} / \mathrm{L}$

1992-05-03 收稿，1992-06-02 收修改稿

* 国家自然科学基金资助项目 
的 $\mathrm{HNO}_{3}$ 溶液, 所用试剂均为分澵纯, 所用水均为去离子水.

2. 银膜制作 在洁净的玻璃基片上, 用化学方法淀积上粗䊁度约为 1000 息的银膜(即 银镜) 作为 SERS 活些衬底 ${ }^{[8]}$.

3. 拉曼测量 在 Spex-1403 谱仪上进行. 激发光源为 $\mathrm{He}-\mathrm{Ne}$ 激光器 (6328 $\mathrm{A}$ ), 通 过一个聚焦透镜 $(f=140 \mathrm{~mm})$ 和一个反射镜使点状光斑以 $45^{\circ}$ 角照射样品. 样品处功率约 为 $30 \mathrm{~mW}$, 双光栅单色仪㖓宽 $400 \mu \mathrm{m}$.

\section{二、结果与讨论}

将新制作的银镜直故入洁净的样品池中, 用取样器取 $0.05 \mathrm{~mol} / \mathrm{L}$ 的 $\mathrm{KSCN}$ 溶液 40 $\boldsymbol{\mu L}$, 滴人样品池中, $5 \mathrm{~min}$ 后测其 SERS 谱. 然后在原样品池内再滴人 $0.05 \mathrm{~mol} / \mathrm{L}$ 的 $\mathrm{KSCN}$ 溶液 $40 \mu \mathrm{L}$ 和 $1.3 \times 10^{-6} \mathrm{~mol} / \mathrm{L}$ 的 $\mathrm{Bi}^{3+}$ 离子溶液 $40 \mu \mathrm{L}, 5 \mathrm{~min}$ 后测其 SERS 谱, 以这样 的步骤,依次得到不同 $\mathrm{Bi}^{3+}$ 离子浓度下, $\mathrm{SCN}^{-} / \mathrm{Ag}$ 系统的 SERS 谱(图 1).

由于实验中使用的溶液含 $\mathrm{NO}_{3}^{-}$离子, 为了确证 $\mathrm{SCN}^{-} / \mathrm{Ag}$ SERS 谱的变化是由 $\mathrm{Bi}^{3+}$ 离 子引起, 而不是由 $\mathrm{NO}_{3}$ 离子引起, 我们进行了如下实验: 选用一片具有大致相同数量级增强 因数 $\left(E_{F}\right)$ 的眼镜, 在 $1900-2300 \mathrm{~cm}^{-1}$ 之间做出 $0.05 \mathrm{~mol} / \mathrm{L} \mathrm{KSCN}$ 溶液的 SERS 谱, 加人 $0.02 \mathrm{~mol} / \mathrm{L}$ 的 $\mathrm{HNO}_{3}$ 溶液（对应上述实验中涉及到的最大 $\mathrm{NO}_{3}^{-}$离子浓度） $20 \mathrm{~min}$ 后的 SERS 谱和再加人 $8.0 \times 10^{-4} \mathrm{~mol} / \mathrm{L} \mathrm{Bi}{ }^{3+}$ 贸子 $5 \mathrm{~min}$ 后的 SERS 谱, 三个谱均画在图 2, 图 2 的 强度轴是图 1 的四分之一, 即图 2 中的谱相对图 1 来说放大了 4 倍. 从图 2 中清楚地看出, $\mathrm{SCN}^{-} / \mathrm{Ag}$ 的 SERS 谱的变化, 确是由 $\mathrm{Bi}^{3+}$ 的加人引起的.

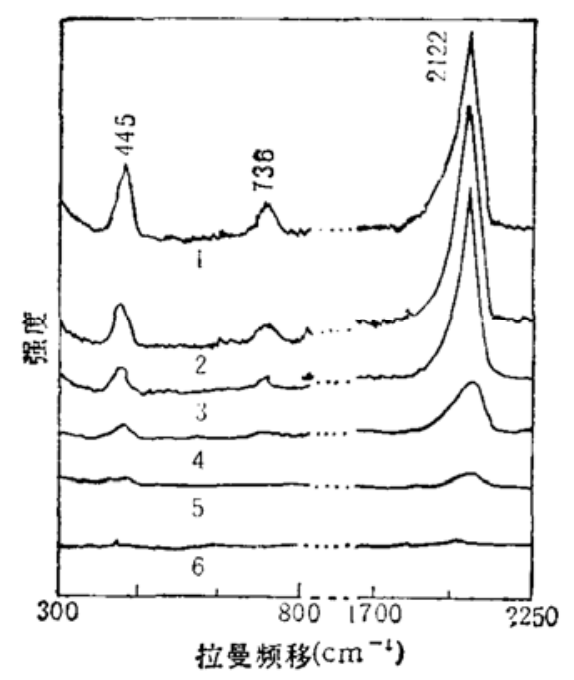

图 1 不同 $\mathrm{Bi}^{3+}$ 浓度下 $\mathrm{SCN}^{-} / \mathrm{Ag}$ 的 $\mathrm{SERS}$ 谱 $1-0.05 \mathrm{~mol} / \mathrm{L} \mathrm{KSCN}$,

$2-0.05 \mathrm{~mol} / \mathrm{L} \mathrm{KSCN}+1.3 \times 10^{-6} \mathrm{~mol} / \mathrm{L} \mathrm{Bi}^{3+}$, $3-0.05 \mathrm{~mol} / \mathrm{L} \mathrm{KSCN}-6.4 \times 10^{-6} \mathrm{~mol} / \mathrm{L} \mathrm{Bi}^{3+}$, $4-0.05 \mathrm{~mol} / \mathrm{L} \mathrm{KSCN}-3.2 \times 10^{-9} \mathrm{~mol} / \mathrm{L} \mathrm{Bi}^{3+}$, $5-0.05 \mathrm{~mol} / \mathrm{L} \mathrm{KSCN}+1.6 \times 10^{-4} \mathrm{~mol} / \mathrm{L} \mathrm{Bi}{ }^{3+}$, $6-0.05 \mathrm{~mol} / \mathrm{L} \mathrm{KSCN}+8.0 \times 10^{-4} \mathrm{~mol} / \mathrm{L} \mathrm{Bi}^{3+}$

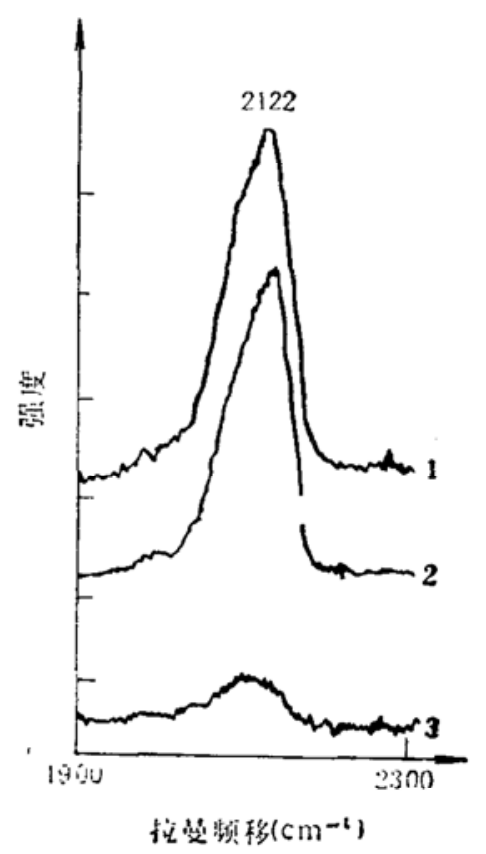

图 $2 \mathrm{SCN}^{-} / \mathrm{Ag}$ 的 SERS 谱 $\left(2122 \mathrm{~cm}^{-1}\right.$ 峰)变化

$1-0.05 \mathrm{~mol} / \mathrm{L} \mathrm{KSCN}$,

$2-0.05 \mathrm{~mol} / \mathrm{L} \mathrm{KSCN}+0.02 \mathrm{~mol} / \mathrm{L} \mathrm{HNO}_{3}$,

$3-0.05 \mathrm{~mol} / \mathrm{L} \mathrm{KSCN}+0.02 \mathrm{~mol} / \mathrm{L} \mathrm{HNO}_{3}$ $+8.0 \times 10^{-+} \mathrm{mol} / \mathrm{L} \mathrm{Bi}^{3+}$ 
我们注意到, $\mathrm{Bi}^{3+}$ 离子的加人没有改变该系统 SERS 蜂的位置, 这说明 $\mathrm{Bi}^{3+}$ 的存在既 没有改变 $\mathrm{SCN}^{-}$的构型, 也没有改变 $\mathrm{SCN}^{-}$与银键接的方式, 这是因为 $\mathrm{Bi}^{3+}$ 不对 $\mathrm{SCN}^{-}$单 独作用, 而作用于 $\left[\mathrm{Ag}(\mathrm{SCN})_{2}\right]^{-}$中的 $\mathrm{Ag}^{+}$离子, 这与文献 [9] 报道的 $\mathrm{Tl}^{+}$对 SERS 的影 响是类似的. 另外, $\mathrm{Bi}^{3+}$ 离子的加人, 使该系统 SERS 谱的峰宽略为变窄, 这一首次观察到 的现象可能是由于 $\mathrm{Bi}^{3+}$ 离子对 $\left[\mathrm{Ag}(\mathrm{SCN})_{2}\right]^{-}$离子的振动驰豫过程产生了影响 ${ }^{[10]}$.

上面的实验现象, 使我们肯定地认为 $\mathrm{Bi}^{3+}$ 离子对 SERS 存在着复杂的影响,一个定性的 作用模型有助于解释观察到的现象: 到达银表面的 $\mathrm{Bi}^{3+}$ 离子, 由银表面提供电子, 被还原为 $\mathrm{Bi}$ 原子, $\mathrm{Bi}$ 原子通过表面上的扩散而接近 $\left[\mathrm{Ag}(\mathrm{SCN})_{2}\right]^{-}$, 并提供电子给 $\mathrm{Ag}^{+\mathrm{t} 111}$, 使 $\mathrm{Ag}^{+}$还 原为 $\mathrm{Ag}^{0},\left[\mathrm{Ag}(\mathrm{SCN})_{2}\right]^{-}$解体, 从而 $\mathrm{SCN}^{-} / \mathrm{Ag}$ 系统 SERS 信号减弱. 在这一过程中 $[\mathrm{Ag}$ $\left.(\mathrm{SCN})_{2}\right]^{-}$的振动无疑会受到影响, 因此, 其峰宽变窄是可以理解的

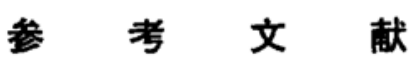

[ 1 ] Fleischman, M., Hendra, P. J. et al., Chem. Phys. Lett., 26(1974), 163.

[2] Aroca, R., Vib. Spectra Struct., 19(1991), 55.

[3] Rubim, J. C., Chem. Phys. Lett., 167(1990), 209.

[4] Temperini, M. L. A. et al., J. Raman. Spectrosc, 22(1991), 301.

[5] Weaver, M. J., Barz, F. er al., Surt. Sci., 125(1983), 409.

[6] Bailey, R. A. et al., Coord. Chem. Rev, 6(1971), 407.

[ 7 ] Gold, H. S., Buck, R. P., J. Raman Spectrosc., 8(1979), 323.

[ 8 ] Mo, Y., Mörke, l., Wachter, P., Surf. Sci., 133(1983), L452.

[9] Furtak, T. E., Roy, D., Phys. Rev. Lett, 50(1983), 1301.

[10] Oxtoby, D. W., J. Phys. Chem., 87(1983), 3028.

[11] Watanabe, T. et al., Chem. Phys. Lent, 102(1983), 565. 